\title{
First direct detection of a Keplerian rotating disk around the Be star $\alpha$ Arae using AMBER/VLTI ${ }^{\star}$
}

\author{
A. Meilland ${ }^{1}$, P. Stee ${ }^{1}$, M. Vannier $2,6,7$, F. Millour ${ }^{2,3}$, A. Domiciano de Souza ${ }^{2,1}$, F. Malbet ${ }^{3}$, C. Martayan ${ }^{4}$, F. Paresce ${ }^{5}$, \\ R.G. Petrov ${ }^{2}$, A. Richichi ${ }^{5}$, and A. Spang ${ }^{1}$ \\ 1 Laboratoire Gemini, UMR 6203 Observatoire de la Côte d'Azur/CNRS, BP 4229, 06304 Nice Cedex 4, France \\ ${ }^{2}$ Laboratoire Universitaire d'Astrophysique de Nice, UMR 6525 Université de Nice, Sophia Antipolis/CNRS, Parc Valrose, \\ 06108 Nice Cedex 2, France \\ 3 Laboratoire d'Astrophysique de Grenoble, UMR 5571 Université Joseph Fourier/CNRS, BP 53, 38041 Grenoble Cedex 9, France \\ ${ }^{4}$ GEPI-Observatoire de Paris-Meudon, UMR 8111 Université Denis Diderot/CNRS, 5 Place Jules Janssen, 92195 Meudon Cedex, \\ France \\ 5 European Southern Observatory, Karl Schwarzschild Strasse 2, 85748 Garching, Germany \\ ${ }^{6}$ European Southern Observatory, Casilla 19001, Santiago 19, Chile \\ 7 Departamento de Astronomia, Universidad de Chile, Chile
}

Received 12 January 2006 / Accepted 1 June 2006

\section{ABSTRACT}

\begin{abstract}
Aims. We aim to study the geometry and kinematics of the disk around the Be star $\alpha$ Arae as a function of wavelength, especially across the $\mathrm{Br} \gamma$ emission line. The main purpose of this paper is to understand the nature of the disk rotation around Be stars.

Methods. We use the AMBER/VLTI instrument operating in the $K$-band, which provides a gain by a factor of 5 in spatial resolution compared to previous MIDI/VLTI observations. Moreover, it is possible to combine the high angular resolution provided with the (medium) spectral resolution of AMBER to study the kinematics of the inner part of the disk and to infer its rotation law.

Results. For the first time, we obtain direct evidence that the disk is in Keplerian rotation, answering a question that has existed since the discovery of the first Be star $\gamma$ Cas by Father Secchi in 1866. We also present the global geometry of the disk, showing that it is compatible with a thin disk and polar enhanced winds modeled with the SIMECA code. We found that the disk around $\alpha$ Arae is compatible with a dense equatorial matter confined to the central region, whereas a polar wind is contributing along the rotational axis of the central star. Between these two regions, the density must be low enough to reproduce the large visibility modulus (small extension) obtained for two of the four VLTI baselines. Moreover, we obtain that $\alpha$ Arae is rotating very close to its critical rotation. This scenario is also compatible with the previous MIDI measurements.
\end{abstract}

Key words. techniques: high angular resolution - techniques: interferometric - stars: emission-line, Be - stars: kinematics stars: individual: $\alpha$ Arae - stars: circumstellar matter

\section{Introduction}

The star $\alpha$ Arae (HD 158 427, HR 6510, B3 Ve), one of the closest ( $d=74$ pc, Hipparcos, Perryman et al. 1997) Be stars, was observed with the MIDI/VLTI instrument at $10 \mu \mathrm{m}$ in June 2003 and its circumstellar environment was unresolved even with the $102 \mathrm{~m}$ baseline (Chesneau et al. 2005, hereafter Paper I). $\alpha$ Arae was a natural choice as first target, due to its proximity, but also its large mid-IR flux and its high infrared excess among other Be stars, e.g. $E(V-L) \sim 1.8$ and $E(V-12 \mu \mathrm{m}) \sim 2.23$. These first IR interferometric measurements indicated that the size of the circumstellar environment was smaller than predicted by Stee (2003) for the $K$-band. The fact that $\alpha$ Arae remain unresolved, but at the same time had strong Balmer emission, put very strong constraints on the parameters of its circumstellar disk. Independently of the model, they have an upper limit of the envelope size in the $N$-band of $\phi_{\max }=4$ mas, i.e., $14 R_{\star}$, if the star is at $74 \mathrm{pc}$ according to Hipparcos parallax or $20 R_{\star}$

* Based on observations collected at the European Southern Observatory, Paranal, Chile, within the science demonstration time programme 074 . A-9026 (A). if the star is at $105 \mathrm{pc}$, as suggested by the model presented in Paper I.

The authors finally propose a scenario where the circumstellar environment remains unresolved due to an outer truncation of the disk by an unseen companion. Nevertheless, this companion would be too small and too far away to have any influence on the Be phenomenon itself.

To study the inner part of this circumstellar truncated disk, we have taken advantage of the higher spatial resolution by observing with the AMBER/VLTI instrument at $2 \mu \mathrm{m}$ in February 2005. It provides a gain by a factor of 5 in spatial resolution compared to MIDI/VLTI observations. In this paper we present these measurements showing, for the first time, a fully resolved circumstellar envelope in the $\mathrm{Br} \gamma$ emission line and a clear signature of a Keplerian rotating disk around $\alpha$ Arae. We also discuss the challenging question of the nature of the geometry of the Be disks, and particularly their opening angle, since it is still an active debate.

Following the Wind Compressed Disk model (WCD) by Bjorkman \& Cassinelli (1993), most authors have considered geometrically thin disks (half opening angle of 2-5 degrees), even 
Table 1. Model parameters for the $\alpha$ Arae central star and its circumstellar environment obtained from Paper I.

\begin{tabular}{cc}
\hline \hline Parameter/result & Value \\
\hline Spectral type & B3Ve \\
$T_{\text {eff }}$ & $18000 \mathrm{~K}$ \\
Mass & $9.6 M_{\odot}$ \\
Radius & $4.8 R_{\odot}$ \\
Luminosity & $5.8 \times 10^{3} L_{\odot}$ \\
Inclination angle $i$ & $45^{\circ}$ \\
Photospheric density $\left(\rho_{\text {phot }}\right)$ & $1.2 \times 10^{-12} \mathrm{~g} \mathrm{~cm}^{-3}$ \\
Photospheric expansion velocity & $0.07 \mathrm{~km} \mathrm{~s}^{-1}$ \\
Equatorial rotation velocity & $300 \mathrm{~km} \mathrm{~s}^{-1}$ \\
Equatorial terminal velocity & $170 \mathrm{~km} \mathrm{~s}^{-1}$ \\
Polar terminal velocity & $2000 \mathrm{~km} \mathrm{~s}^{-1}$ \\
Polar mass flux & $10^{-9} M_{\odot} \mathrm{year}^{-1} \mathrm{sr}^{-1}$ \\
$m 1$ & 0.3 \\
$m 2$ & 0.45 \\
$C 1$ & 30 \\
Mass of the disk & $2.3 \times 10^{-10} M_{\odot}$ \\
Mass loss & $6.0 \times 10^{-7} M_{\odot} \mathrm{year}^{-1}$ \\
\hline
\end{tabular}

if Owocki et al. (1996) have found that the equatorial wind compression effects are suppressed in any radiatively driven wind models for which the driving forces include a significant part from optically thick lines. Moreover, they found that gravity darkening effects can lead to a reduced mass loss, and thus a lower density in the equatorial regions. A wind compression effect is, however, not required to produce the small opening angle of the disk. The investigation of accretion disks has shown that disks in hydrodynamical equilibrium and Keplerian rotation will not have much larger opening angles, since their scale height is governed by vertical gas pressure only. For a disk to be thicker, either additional mechanisms have to be assumed, or it might not be in equilibrium at the radii in question (Bjorkman \& Carciofi 2004).

On the other hand, Stee et al. $(1995,1998)$ claimed that Be disks must be more ellipsoidal to reproduce the strong IR excess observed and interpret the possibility for a $\mathrm{Be}$ star to change its spectral type from $\mathrm{Be}$ to $\mathrm{B}$, and more rarely $\mathrm{Be}$ to Be-Shell type, i.e., where the disk is dense enough to produce a strong "shell" absorption.

In the following we adopt, as a starting point, the same parameters for the modeling of $\alpha$ Arae, e.g., the central star and its circumstellar envelope used in Paper I and summarized in Table 1 . Following the polarization measurements $\mathrm{Pl} \approx 0.6 \%$ and Position Angle (PA) of $172^{\circ}$ by McLean \& Clarke (1979) and Yudin (2001), the disk major-axis orientation is expected to be at about PA $\approx 82^{\circ}$ (Wood et al. 1996a,b; Quirrenbach et al. 1997). Assuming a stellar radius of $4.8 R_{\odot}$ and an effective temperature $T_{\text {eff }}=18000 \mathrm{~K}$, the photospheric angular diameter is estimated to be 0.7 mas (Cohen et al. 1998; Chauville et al. 2001). For the distance of $74 \mathrm{pc}$ and a baseline of $60 \mathrm{~m}$ at $2 \mu \mathrm{m}$, Stee (2003) predicts the visibility of $\alpha$ Arae to be lower than 0.2 , i.e., fully resolved.

The paper is organized as follows. In Sects. 2 and 3 we present the interferometric AMBER observations and the data reduction. In Sect. 4 we try to obtain a first estimate of $\alpha$ Arae's envelope geometry using very simple "toy" models. Section 5 briefly describes the SIMECA code. In Sect. 6 we present the best model we obtain with SIMECA that fits both the $\mathrm{Br} \gamma$ line and the visibility modulus and phase as a function of wavelength, which allows us to infer the disk kinematics and its rotational velocity. Finally, Sect. 8 draws the conclusions from these first spectrally resolved interferometric measurements of a Be star at $2 \mu \mathrm{m}$.

\section{AMBER/VLTI observations}

Our observations of $\alpha$ Arae were acquired during a Science Demonstration Time (SDT) run, on the nights of February 23-24, 2005, using the AMBER instrument (Petrov et al. 2007) on the VLTI in medium-resolution mode $(R=1500)$. On the night of February 24, the observations were made with two UT telescopes, i.e., one interferometric baseline only, and consist of six exposure files, each containing 500 frames of $100 \mathrm{~ms}$. On the following night, three telescopes were used and data of three baselines were taken in a series of three exposure files and another series of two, each containing 500 frames of $70 \mathrm{~ms}$. Without a fringe tracker, the integration time per frame must be short enough to minimize the smearing of the fringe visibility due to the beams jitter, while still having enough photons over the elementary exposure. Its specific value is chosen depending on the atmospheric conditions. Immediately after observing $\alpha$ Arae, a nearby calibrator object was observed.

\section{Reduction of the interferometric data}

The data has been reduced using the "ammyorick" package developped by the AMBER consortium. The principles of the AMBER data reduction have been described by Tatulli et al. (2007). In addition to the tools furnished by the default package, some specific treatments have been added to reach an optimal precision on the interferometric observables. The various steps are the following. For each of the individual exposure frames, the complex visibilities are extracted from the various interferometric channels and calibrated using the photometric channels and some internal calibration files. The piston $p$ between the beams is first estimated from the slope of the fringes with wavelength, and a correction phaser is applied on each frame. From this, the color-differential phase and visibility are calculated, for a given spectral channel $\lambda$, with respect to a set of reference channel(s). We chose the reference channel to occur in the whole spectral bandwidth except the considered channel $\lambda$. Due to the jitter of the beams and to the subsequent variations of output flux after the optical fibers of AMBER, not all the frames contain good quality fringes. A selection and weighing of the best frames above a given threshold is made based on a fringe quality criterion. This yields our color-differential estimators of phase and visibility with an optimal precision. For both the science source and its calibrator, the statistical deviation of the differential phases over the frames series is about $0.01 \mathrm{rad}$ per spectral channel. The deviation of the normalized differential visibility is about $0.5 \%$. These numbers are similar for all the baselines.

The scaling of the differential visibilities to "absolute" visibilities is done through a best adjustment of the "science" data to that measured on the calibrator star, whose theoretical visibility is known. For this, the histogram of the squared visibilities from all the science frames (with enough flux above the detector noise) is fitted, by a scaling factor, to the histogram of the calibrator star. With the data we have, this fit has been done globally, by integrating the spectral channels together for each frame. Therefore, some possible bias between the spectral channels would not be suppressed by the calibration process.

In the present case, the observations of the nominal calibrator for $\alpha$ Arae were of poor quality, and another calibrator, at some distance on the sky $\left(\approx 45^{\circ}\right)$, had to be used. This does not 
Table 2. Observation log, with the projected baseline lengths and angles. The diameter for the calibrator used is taken from the CHARM catalogue (Richichi \& Percheron 2002).

\begin{tabular}{ccccccc}
\hline \hline Date & Obs. time & Baselines & Length & PA & Calibrator & $\phi_{\text {cal. }}$ \\
\hline \multirow{2}{*}{$24 / 12 / 2005$} & (UTC) & & $(\mathrm{m})$ & $\left({ }^{\circ}\right)$ & & (mas) \\
& $08: 46$ & UT2-4 $\left(B_{0}\right)$ & 80.9 & 39 & HD124454 & $1.52 \pm 0.02$ \\
& & UT2-3 $\left(B_{1}\right)$ & 46.4 & 19 & & \\
$25 / 12 / 2005$ & $09: 40$ & UT3-4 $\left(B_{2}\right)$ & 52.5 & 81 & & \\
\hline
\end{tabular}

seem to affect the calibration, though. The scaling fit between the histograms of the science and calibrator objects results in an error of about $5 \%$ on the visibilities, on every baseline.

\section{Study of the envelope morphology using simple models}

In this section we present the new AMBER data with the previous MIDI data already presented in Paper I to obtain a first estimate of $\alpha$ Arae's envelope geometry using very simple "toy" models.

\subsection{Envelope extension}

\subsubsection{Extension in the continuum at $2.1 \mu \mathrm{m}$}

Assuming that the visibility we measure in the continuum, $V_{\mathrm{c}}$, is only due to the central star and its circumstellar disk we can write:

$V_{\mathrm{c}}=\frac{V_{\mathrm{ec}} F_{\mathrm{ec}}+V_{\star \mathrm{c}} F_{\star \mathrm{c}}}{F_{\mathrm{tot}}}$,

where $V_{\mathrm{ec}}$ and $F_{\mathrm{ec}}$ are, respectively, the envelope visibility and flux in the continuum, $V_{\star c}$ and $F_{\star c}$ the star visibility and flux in the continuum, and $F_{\text {tot }}=F_{\text {ec }}+F_{\star c}$. Since $V_{\mathrm{c}}$ is the measured visibility, to only estimate the envelope visibility, $V_{\text {ec }}$, we can rewrite Eq. (1):

$V_{\mathrm{ec}}=\frac{V_{\mathrm{c}} F_{\mathrm{tot}}-V_{\star \mathrm{c}} F_{\star \mathrm{c}}}{F_{\mathrm{ec}}}$.

The total flux is normalized, i.e. $F_{\text {tot }}=F_{\mathrm{ec}}+F_{\star \mathrm{c}}=1$. Since the star is almost unresolved, $0.5<\phi_{\star}<0.7$ mas, which corresponds to $0.98<V_{\star c}<0.99$ for the longest baseline at $2.1 \mu \mathrm{m}$, we assume in the following that $V_{\star \mathrm{c}}=1$. To estimate $V_{\mathrm{ec}}$, we still have to determine the star and envelope contributions to the $2.1 \mu \mathrm{m}$ flux continuum. Since the envelope continuum flux in the visible and in the UV is negligible, we have fitted the blue part of the SED using a $T_{\text {eff }}=18000 \mathrm{~K}$ blackbody for the central star in order to deduce the envelope emission at larger wavelengths. At $2.1 \mu \mathrm{m}$ we found that the star emission is still $\sim 1.5$ larger, i.e., 0.44 mag brighter, than the envelope contribution; thus, following $F_{\star \mathrm{c}}=1.5 F_{\mathrm{ec}}$, we obtain $F_{\star \mathrm{c}}=0.6$ and $F_{\text {ec }}=0.4$. Equation (2) can now be rewritten as:

$V_{\mathrm{ec}}=2.5 V_{\mathrm{c}}-1.5$.

The continuum visibilities and envelope extensions we obtained following Eq. (3), assuming a uniform disk model, are given in Table 3.
Table 3. Visibilities measured in the continuum at $2.1 \mu \mathrm{m}\left(V_{\mathrm{c}}\right)$ and the $\operatorname{Br} \gamma$ line $\left(V_{\mathrm{r}}\right)$, and deduced for the envelope contribution only, using a uniform disk model, in the continuum $\left(V_{\mathrm{ec}}\right)$ and in the $\operatorname{Br} \gamma$ line $\left(V_{\mathrm{er}}\right)$ respectively. The corresponding angular diameters obtained for the envelope are given in the continuum $\phi_{\mathrm{ec}}$ and in the line $\phi_{\mathrm{er}}$.

\begin{tabular}{c|cccc}
\hline \hline Base $\mathrm{N}^{\circ}$ & 0 & 1 & 2 & 3 \\
\hline Description & UT2-4 & UT2-3 & UT3-4 & UT2-4 \\
Length (m) & 80.9 & 46.4 & 52.5 & 84.6 \\
PA $\left(^{\circ}\right)$ & 39 & 19 & 81 & 52 \\
$V_{\mathrm{c}}$ & 0.84 & 0.80 & 0.72 & 0.73 \\
$V_{\mathrm{r}}$ & 0.63 & 0.70 & 0.59 & 0.55 \\
$V_{\mathrm{ec}}$ & 0.60 & 0.50 & 0.30 & 0.33 \\
$V_{\mathrm{er}}$ & 0.21 & 0.50 & 0.33 & 0.19 \\
$\phi_{\mathrm{ec}}$ (mas) & $3.3 \pm 1.2$ & $6.8 \pm 1.6$ & $7.6 \pm 1.4$ & $4.5 \pm 0.9$ \\
$\phi_{\mathrm{er}}$ (mas) & $5.3 \pm 2.0$ & $6.8 \pm 1.4$ & $7.3 \pm 2.1$ & $5.2 \pm 1.2$ \\
\hline
\end{tabular}

\subsubsection{Extension in the $\mathrm{Br} \gamma$ line}

We can define the same equation as Eq. (2) for the envelope visibility in the following $\operatorname{Br} \gamma$ line:

$V_{\mathrm{er}}=\frac{V_{\mathrm{r}} F_{\mathrm{r}}-V_{\mathrm{c}} F_{\mathrm{tot}}}{F_{\mathrm{er}}}$

where $V_{\mathrm{r}}$ and $F_{\mathrm{r}}$ are, respectively, the measured visibility and flux in the $\mathrm{Br} \gamma$ line. $V_{\mathrm{c}}$ and $F_{\text {tot }}$ are the quantities previously defined, and $V_{\mathrm{er}}$ and $F_{\mathrm{er}}$ are the visibility and flux only due to the envelope, i.e., without the stellar contribution. We obtain $F_{\text {er }}=$ 0.5 and $F_{\mathrm{r}}=1.5$ at the center of the line using the Br $\gamma$ emission line profile. The values of $V_{\mathrm{er}}$ we obtain using Eq. (4), and assuming a uniform disk model, are given in Table 3.

Globally, we obtain that, using a simple uniform disk model for each measurement, the $\operatorname{Br} \gamma$ emission in the line and the nearby continuum at $2.1 \mu \mathrm{m}$ have the same extension, about 7-8 mas along the UT2-UT3 $\left(\mathrm{B}_{1}\right)$ and UT3-UT4 $\left(\mathrm{B}_{2}\right)$ baselines, and about 5 mas for UT2-UT4 $\left(B_{3}\right)$ and $\sim 4$ mas for UT2-UT4 $\left(\mathrm{B}_{0}\right)$ (which was measured at a slightly different PA).

\subsection{Comparison between MIDI and AMBER extensions}

To compare the extensions we obtain with AMBER at $2.1 \mu \mathrm{m}$ (see Table 3) with the MIDI data presented in Paper I, we have calculated the envelope extension in the continuum between 7 and $13 \mu \mathrm{m}$ following Eq. (2), assuming a uniform disk for each measurement. Unfortunately, these MIDI observations were conducted under unfavorable conditions with thin cirrus passing, and the data were reduced with one of the first versions of the software available. This led to error bars between $8 \%$ and $18 \%$, insufficient to measure a significant change in the visibility and thus in the diameter between 7 and $13 \mu \mathrm{m}$. Despite these limitations, these measurements have given a rough upper limit value of the envelope extension around 10 mas in the $N$-band (see Fig. 1) and are compatible with our AMBER measurements. 


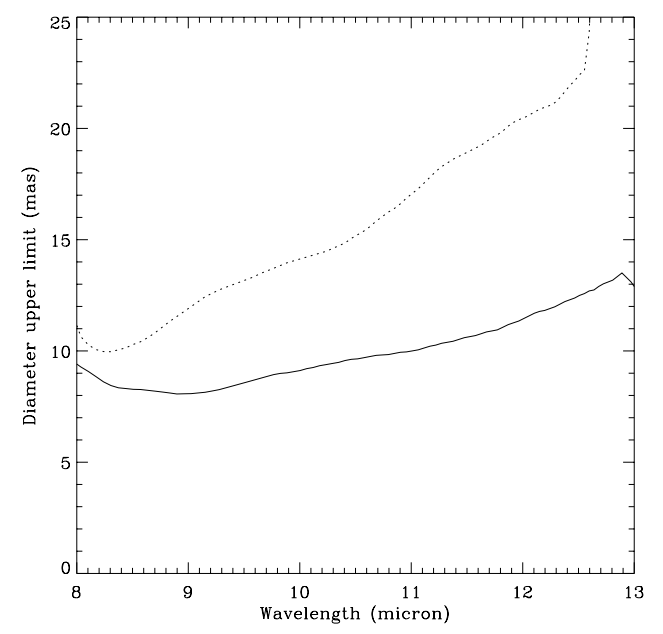

Fig. 1. $\alpha$ Arae (unresolved star + uniform disk) model upper limit diameters (in mas), as a function of wavelength, from the 2005 MIDI data described in Paper I for the June 16 (solid line) and June 17 nights (dotted line). Note that the large values obtained for larger wavelengths are essentially due to poor calibration and large error bars rather than a true physical effect.

Moreover, the extension of the $\alpha$ Arae circumstellar environment seems to be almost independent of the wavelength, which puts strong constraints on the density law within the envelope. The large upper limit values obtained for larger wavelengths are essentially due to poor calibration and large error bars rather than a true physical effect. For "classical" Be stars models, where the density is slowly and regularly decreasing as a function of the distance from the central star, we predict an increasing size as a function of wavelength, as shown by Stee (2001). One possible way of keeping the same angular size between 2 and $13 \mu \mathrm{m}$ may be a disk truncation by an external physical effect, as proposed in Paper I.

Using FEROS spectroscopic data and the fact that their MIDI/VLTI data are showing a nearly unresolved envelope, the authors propose a possible disk truncation by an unseen companion at a radius of about $154 R_{\odot}$, assuming a circular orbit for the companion with negligible mass. With $R_{\star}=4.8 R_{\odot}$, this corresponds to about 32 stellar radii, i.e., 6.4 mas, which is in agreement with their estimate based on the MIDI/VLTI data for a disk truncated at $25 R_{\star}$, i.e., 4 mas, somewhat smaller than the companion orbit. This is also in agreement with what we have obtained with AMBER, i.e., an envelope extension at $2.1 \mu \mathrm{m}$ around 7 mas, which may appear larger than the 4 mas they obtained. Nevertheless, we must keep in mind that they estimate their extensions using a uniform disk for the star + disk emission, whereas we use an unresolved star + a uniform disk only for the circumstellar envelope leading to a larger size.

Finally, we have verified that our best model described in Sect. 6 was compatible with the MIDI data obtained in 2003, showing a nearly unresolved envelope.

\subsection{Envelope geometry}

The envelope extensions presented in Table 3 are very sensitive to the sky-plane baseline orientation. This is particularly obvious from Fig. 2 where we have plotted $\alpha$ Arae's (unresolved star + uniform disk) model diameters as a function of the sky-plane baseline position. In the following sections, we present very simple models to constrain the geometry of the circumstellar envelope by fitting the data obtained (see Fig. 2). Note also that

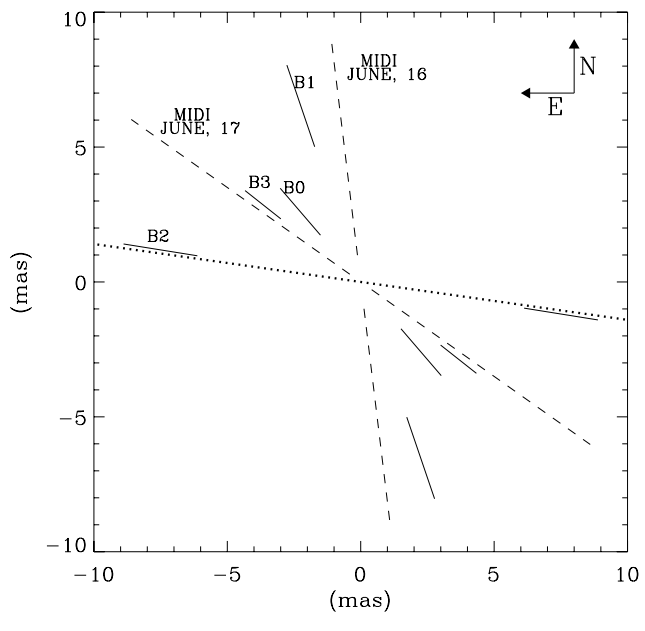

Fig. 2. $\alpha$ Arae (unresolved star + uniform disK) model diameters's (in mas) dependence on the baseline position angle. Solid line: AMBER measurements in the continuum at $2.1 \mu \mathrm{m}$. Dashed lines: MIDI measurements. The length of the plot corresponds to the values of the error bars (note the very large error bars for the MIDI data). The dotted line is the direction of the majoraxis of the envelope estimated from polarization measurements $\left(\mathrm{PA}=172^{\circ}\right)$ obtained by McLean \& Clarke (1979) and Yudin et al. (1998).

here we present models for the light distribution on the sky, not "physical" models, which will be presented hereafter.

\subsubsection{Equatorial disk perpendicular to the polarization}

Our starting point is the equatorial plane position of the circumstellar environment of $\alpha$ Arae deduced from the polarization measurement done by McLean \& Clarke (1979) and Yudin et al. (1998). The polarization angle measured is $\mathrm{PA}=172^{\circ}$, and any flattened envelope model should have a semi-major axis perpendicular to this direction, i.e., around $82^{\circ}$.

In the following, we consider simple axisymmetric disk models, presented in Fig. 3, with 3 free parameters:

1. the inclination angle $(i)$ between the observer and the polar axis $\left(i=0^{\circ}\right.$ corresponds to pole-on);

2. the opening angle $(\alpha)$ of the disk;

3 . the disk extension $(a)$ in mas.

The shape of the projection of the disk onto the skyplane depends only on the two first free parameters, i.e., $i$ and $\alpha$. For all these "toy" models, the observer is on the right for the 4 upper pictures in Fig. 3. The corresponding projections into the skyplane with the interferometric data points from MIDI and AMBER overplotted are the central pictures, whereas " $3 \mathrm{D}$ artist views" are plotted in the lower row for each model.

The ratio between the projected majoraxis $(a)$ and the minoraxis $(b)$ of the envelope is given by:

$\frac{a}{b}=\frac{1}{\cos i+2 \sin \frac{\alpha}{4} \sin \left(i-\frac{\alpha}{4}\right)}$,

we assume that the majoraxis of the envelope is oriented at PA = $82^{\circ}$ (i.e., perpendicular to the polarization angle). Since $B_{2}$ is oriented at $81^{\circ}$, it is supposed to be a good estimate of the disk major-axis extension. In Fig. 4 we plotted the ratio $a / b$ as a function of the disk opening angle for different inclination angles between $35^{\circ}$ and $60^{\circ}$. This angle range was determined following the inclination angle of $45^{\circ}$ for $\alpha$ Arae found from the fit of the circumstellar $\mathrm{H} \alpha, \mathrm{H} \beta$, and $\mathrm{P} \beta$ emission lines in Paper I. 

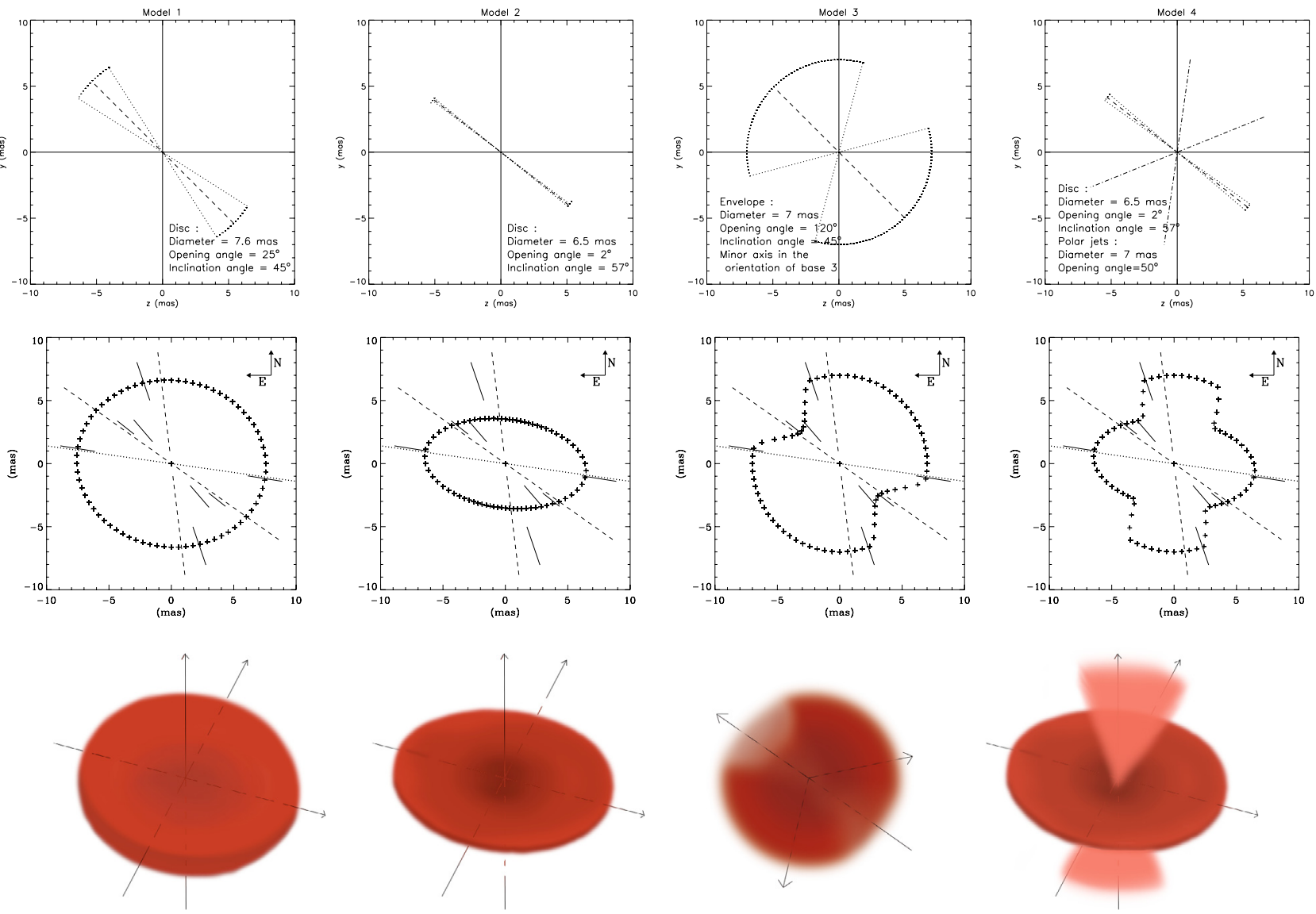

Fig. 3. "Toy" models used to fit the AMBER and MIDI measurements. The 4 upper pictures are a cut of the circumstellar disk in a plane defined by the observer line of sight and the stellar rotational axis (the observer is on the right for each picture); the corresponding projections into the skyplane with the interferometric data points from MIDI and AMBER over-plotted are the central pictures, whereas a "3D artist view" is plotted into the lower row for each model.



Fig. 4. Flatness ratio of the projected envelope versus opening angle calculated with our simple disk model for different inclination angles. Horizontal lines show flatness from models 1 and 2.

From Fig. 4, it is obvious that a precise determination of the inclination angle is mandatory to obtain an accurate opening angle estimation.

Our first disk model 1 with $i=45^{\circ}, \alpha=25^{\circ}$, and $a=7.7$ mas corresponds to a projected ellipse with a $a / b$ ratio of 1.2 . The agreement with the observed data is good, except for the $\mathrm{B}_{3}$ and
$\mathrm{B}_{0}$ baselines, which present a smaller extension than predicted. To fit the data for the $\mathrm{B}_{3}$ and $\mathrm{B}_{0}$ baselines, we define a model 2 with $i=57^{\circ}, \alpha=2^{\circ}$, and $a=6.5$ mas, i.e., smaller and very thin compared to model 1 . In this latter case, the $a / b$ ratio is 1.85 , which allows us to fit the $\mathrm{B}_{2}$ and $\mathrm{B}_{3}$ baselines and MIDI data, but not the $\mathrm{B}_{0}$ and $\mathrm{B}_{1}$ baselines. As shown in Fig. 4, model (2), with a very thin disk, is not compatible with an inclination angle of $45^{\circ}$, and we were obliged to use a larger inclination of $57^{\circ}$. From Fig. 4, we can see that for an inclination angle of $45^{\circ}$, the largest $a / b$ ratio for an extremely thin disk $\left(\alpha \sim 0^{\circ}\right)$ is only 1.41 , i.e., $\sqrt{2}$. Moreover, changing the inclination angle will change the shape of the $\mathrm{H} \alpha, \mathrm{H} \beta$, and $\mathrm{P} \beta$ line profiles, which were used to infer the value of $45^{\circ}$ obtained in Paper I. Finally, using a simple disk model with various extensions, opening and inclination angles failed to simultaneously fit the AMBER and MIDI data, especially for the $\mathrm{B}_{0}$ and $\mathrm{B}_{3}$ baselines, which show a smaller extension along the $\mathrm{PA}=39^{\circ}$ and $52^{\circ}$ skyplane orientation.

\subsubsection{Polar-axis along the $B_{3}$ baseline orientation}

Since the extensions obtained along the $\mathrm{B}_{0}$ and $\mathrm{B}_{3}$ baselines are the smallest ones, we may consider that these baselines are close to the minoraxis of the envelope (i.e., along the stellar polaraxis). Thus, our model 3 with $i=45^{\circ}, \alpha=120^{\circ}$, and $a=7.7$ mas is a very thick disk, as shown in Fig. 4 with the polar axis close to $B_{0}$ and $B_{3}$, but thick enough to go through the $B_{1}$ and $B_{2}$ data points. This model is, in fact, very similar to the one presented in Paper I, but in this case it maybe difficult to obtain a polarization 
large enough to be measured with a $\mathrm{PA}=172^{\circ}$, since the majoraxis of this "disk" is not perpendicular to the polarization direction. Nevertheless, the polarization was measured in the visible, and since it originates from the inner part of the disk, this model cannot be totally excluded.

\subsubsection{Equatorial disk + polar enhanced winds}

One of the shortcomings of the previous models is that they cannot simultaneously reproduce the two main envelope characteristics: the polarization angle at $\mathrm{PA}=172^{\circ}$ and the smallest extension along the $\mathrm{PA}=39^{\circ}$, whereas for the other AMBER baselines, the disk is clearly more extended.

Our last simple model, model 4 tries to take these observational characteristics into account by considering an equatorial disk oriented perpendicular to the polarization angle and flattened enough to reproduce the difference between the extension measured along the $\mathrm{B}_{0}$ and $\mathrm{B}_{2}$ directions. Moreover, to fit the extensions measured for the $\mathrm{B}_{1}$ baseline, we have added polar enhanced winds perpendicular to the disk, as shown in Fig. 3. Thus, it is also possible to fit all the interferometric MIDI and AMBER data points in the sky plane. Nevertheless, due to the fact that the disk is geometrically very thin $\left(\alpha=2^{\circ}\right)$, and for the same reasons already explained for our model 2 , the inclination angle must be around $57^{\circ}$. Moreover, the polar enhanced winds must be very extended in latitude (with an opening angle about $50^{\circ}$ ), and dense enough to fit the $\mathrm{B}_{1}$ measurement.

\subsection{Conclusion about the "toy story"}

Finally it seems that using very simple "toy" models, we failed to reproduce the different extensions of the envelope as a function of the baseline projection onto the skyplane. Thus, in the following section, we will use our model 4 , i.e., geometrically thin disk + polar enhanced winds, which was the one with the better agreement with both AMBER data and polarization direction, as a starting point. Moreover, the formation of the geometrically very thin disk, with a few degrees for the opening angle, seems to be the best scenario up to now, at least for the central Keplerian disk around Be stars.

\section{The SIMECA code: a brief description}

To constrain the physical parameters of the circumstellar environment of $\alpha$ Arae, we used the SIMECA code. This code, described in previous papers (see Stee \& Araùjo 1994; Stee et al. 1995; Stee \& Bittar 2001), has been developed to model the environment of active hot stars. SIMECA computes line profiles, Spectral Energy Distributions (SEDs), and intensity maps, which can be directly compared to high angular resolution observations. The envelope is supposed to be axisymmetric with respect to the rotational axis. No meridional circulation is allowed. We also assume that the physics of the polar regions is well represented by a CAK type stellar wind model (Castor et al. 1975), and the solutions for all stellar latitudes are obtained by introducing a parameterized model, which is constrained by the spectroscopic and interferometric data. The inner equatorial region is dominated by rotation, therefore being quasi-Keplerian. The ionization-excitation equations are solved for an envelope modeled in a $410 \times 90 \times 71$ cube.

The populations of the atomic levels are strongly altered by non-LTE conditions from their $L T E$-values. To calculate them, we start with the LTE populations for each level, and then

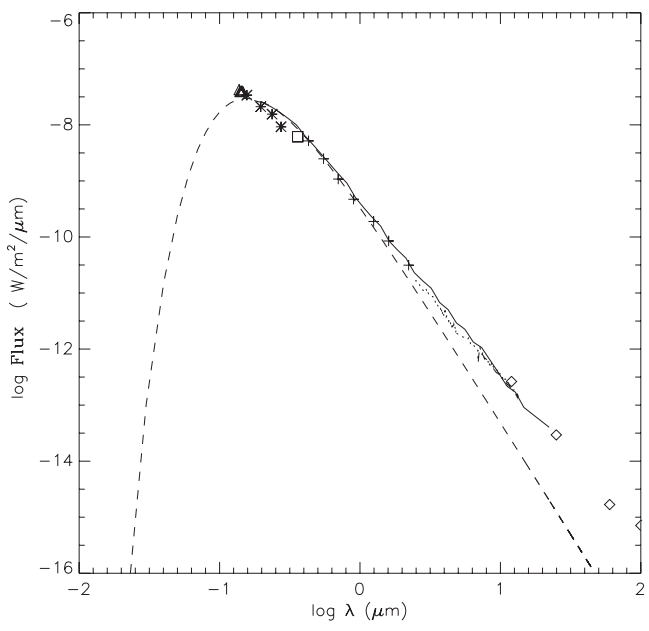

Fig. 5. $\alpha$ Arae's Spectral Energy Distribution (SED) from various sources in the literature (see text). Dashed line: emission from the central star only assuming a blackbody with $R_{\star}=4.8 R_{\odot}, T_{\text {eff }}=18000 \mathrm{~K}$, and $d=105 \mathrm{pc}$. Fit of the SED with SIMECA, taking into account the envelope free-free and free-bound contribution (solid line) between 0.3 and $20 \mu \mathrm{m}$.

compute the escape probability of each transition, obtaining updated populations. By using these populations as input values for the next step, we iterate until convergence. The basic equations of the SIMECA code are given in detail by Stee et al. (1995).

To take into account the photospheric absorption line, we assume the underlying star to be a normal B3 V star with $T_{\text {eff }}=$ $18000 \mathrm{~K}$ and $R=4.8 R_{\odot}$, and synthesize the photospheric line profiles using the SYNSPEC code by Hubeny (Hubeny 1988; Hubeny \& Lanz 1995). The resulting line profile is broadened by solid-body rotation and might be further altered by absorption in the part of the envelope in the line of sight towards the stellar disk.

\section{Using SIMECA for the modeling of $\alpha$ Arae circumstellar environment}

\subsection{Fit of the SED}

Thanks to the previous study, we now have a good starting point for the estimation of the global geometry of the disk around $\alpha$ Arae. Nevertheless, we want to use the SIMECA model already described in Paper I and in Sect. 3 to obtain a more physical scenario for this star. Thus, to obtain a SED that can be used to constrain our model, we have collected photometric measurements from the largest spectral range available, i.e., from UV to mid-IR. We used UV measurements from Jamar (1976) and Thompson (1978), the $U$ magnitude from Johnson 1966, BVRIJHK magnitudes from Ducati 2002, ISO data, MIDI SED measurements (2003) between 7-13 $\mu \mathrm{m}$, and IRAS data. Of course, using non simultaneous data for a variable star may be questionable, but this is a good starting point, especially for the shorter wavelengths related to the central star, which is supposed to be stable, contrary to the circumstellar disk, which may appear and vanish with a typical timescale of a decade, and should drastically modify the IR excess of the SED.

The SED we obtain from these various sources is plotted in Fig. 5. The SED is dominated by the emission of the central star for wavelengths smaller to $1 \mu \mathrm{m}$, which is assumed to be a blackbody with the following parameters from Paper I: $R_{\star}=4.8 R_{\odot}$, $T_{\text {eff }}=18000 \mathrm{~K}$, and $d=105$ pc. In Paper I, we already 
discussed the fact that we were obliged to use a distance of $105 \mathrm{pc}$ instead of the $74 \mathrm{pc}$ obtained from Hipparcos measurements to fit the SED. Without considering any reddening, and keeping the Hipparcos distance, the radius of $\alpha$ Arae would be unrealistically low (below $3.5 R_{\odot}$ ) or the photosphere unrealistically cold $\left(T_{\text {eff }} \sim 15000 \mathrm{~K}\right)$, thus we use the $105 \mathrm{pc}$ determined from Paper I in the following. The free-free and freebound emissions from the envelope produce an IR excess and dominate the SED for wavelengths larger than $\sim 1 \mu \mathrm{m}$, but the envelope remains optically thin. This emission depends mostly on the number of free electrons and on the temperature law in the circumstellar envelope. As the envelope is almost fully ionized, we can consider that the global IR excess is only proportional to the mass of the disk. In Fig. 5, we present our best fit of the SED, using a temperature law in the envelope with $T(r) \propto r^{-3 / 4}$, and a mass of the envelope of $4.1( \pm 0.5) \times 10^{-10} M_{\odot}$.

The mass of the disk in SIMECA depends on six parameters. Three of them are related to the stellar mass loss: mass flux at the pole, $C_{1}$, and $m_{1}$. The three other ones are related to the envelope kinematics: terminal velocity at the pole, at the equator, and $m_{2}$.

The mass flux in SIMECA is given by:

$\Phi(\theta)=\Phi_{\text {pole }}\left[1+(C 1-1) \sin ^{m 1}(\theta)\right]$,

where $m 1$ is the first free parameter, which describes the variation of the mass flux from the pole to the equator, and $C 1$ is the ratio between the equatorial and polar mass flux:

$$
C 1=\frac{\Phi_{\mathrm{eq}}}{\Phi_{\mathrm{pole}}}
$$

The expansion velocity field is given by:

$v_{\mathrm{r}}(r, \theta)=V_{\mathrm{o}}(\theta)+\left[V_{\infty}(\theta)-V_{\mathrm{o}}(\theta)\right]\left(1-\frac{R}{r}\right)^{\gamma}$.

We used $\gamma=0.86$ which is a typical value for early Be stars (Poe \& Friend 1986; Araújo \& Freitas Pacheco 1989; Owocki et al. 1994), with

$V_{\mathrm{o}}(\theta)=\frac{\Phi(\theta)}{\rho_{0}}=\frac{\Phi_{\text {pole }}\left[1+(C 1-1) \sin ^{m 1}(\theta)\right]}{\rho_{0}}$.

The second free parameter $m 2$ is introduced in the expression of the terminal velocity as a function of the stellar latitude:

$V_{\infty}(\theta)=V_{\infty}($ pole $)+\left[V_{\infty}(\right.$ eq $)-V_{\infty}($ pole $\left.)\right] \sin ^{m 2}(\theta)$.

The shape of the terminal velocity law as a function of the stellar latitude, as well as the rotational velocity for various stellar radii are plotted in Fig. 7.

Finally the density distribution in the envelope is given by the equation of mass conservation (see Fig. 6):

$\rho(r, \theta)=\frac{\Phi(\theta)}{\left(\frac{r}{R}\right)^{2} v_{\mathrm{r}}(r, \theta)}$.

The mass of the disk is obtained by integrating Eq. (11). It is possible to obtain a combination of these six parameters that fit the SED, but the solution may, of course, not be unique. To discriminate between all the possible solutions, we need to put more constraints on our modeling, namely, try to fit the visibility measurements in the lines and in the continuum for the various VLTI baseline orientations.

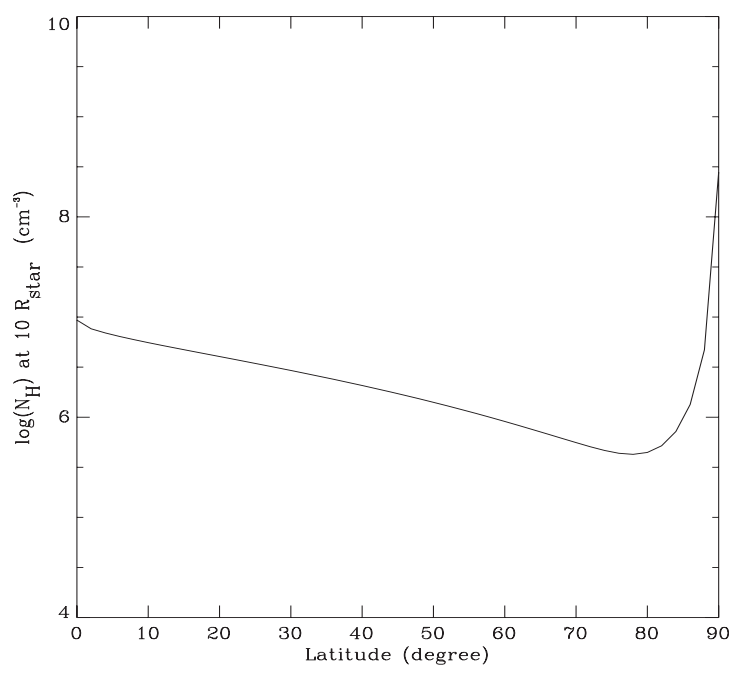

Fig. 6. Example of the SIMECA density distribution as a function of the stellar latitude from the pole $\left(\theta=0^{\circ}\right)$ to the equator $\left(\theta=90^{\circ}\right)$ at $10 R_{*}$.
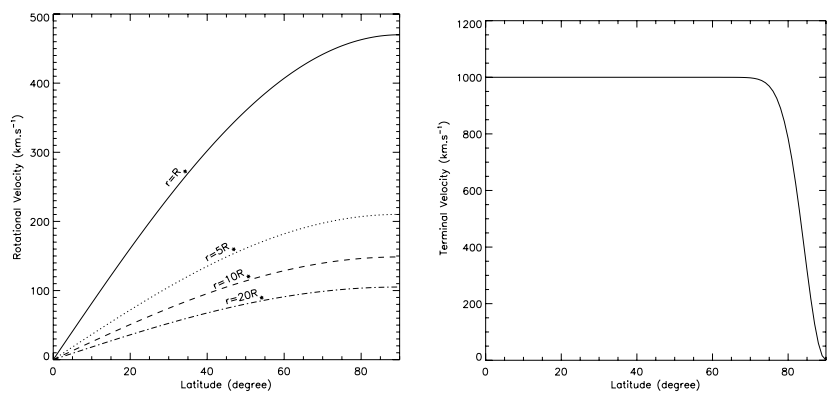

Fig. 7. Left: rotational velocities as a function of the stellar latitudes from the pole $\left(\theta=0^{\circ}\right)$ to the equator $\left(\theta=90^{\circ}\right)$ at various stellar radii (over-plotted on the graph). Right: terminal velocity as a function of the stellar latitudes.

\subsection{Visibility modulus in the continuum}

To fit the visibility measurements described in Sect. 4.2, we simulate a thin disk + polar enhanced winds with SIMECA with a dense equatorial matter confined in the central region, in addition to a polar wind contributing along the rotational axis of the central star. Between these two regions, the density must be low enough to reproduce the large visibility modulus (small extension) obtained for the $\mathrm{B}_{0}$ and $\mathrm{B}_{3}$ VLTI baselines. Since $\rho \propto \frac{\phi}{v_{r}}$ (see Eq. (11)), we can build a model satisfying the above conditions by tuning both the mass flux and the expansion velocity in the envelope. The expansion velocity at the equator should be very small, i.e., a few $\mathrm{km} \mathrm{s}^{-1}$, whereas at the pole it can reach larger values up to $500-2000 \mathrm{~km} \mathrm{~s}^{-1}$. The density ratio between the equator and the pole must be around 10-100 to fit the data. The parameters we obtain for our best model are given in Table 4 , and the $2.15 \mu \mathrm{m}$ intensity map obtained in the continuum is plotted in Fig. 8. The largest discrepancies between these parameters and those from Paper I are that the star is now rotating close to its critical velocity $\left(v=470 \mathrm{~km} \mathrm{~s}^{-1}\right.$, the disk is very thin but denser (by a factor 10)) and the expansion velocity is only $1 \mathrm{~km} \mathrm{~s}^{-1}$. The continuum map is seen with an inclination angle of $55^{\circ}$, and the central bright region is the flux contribution from the thin equatorial disk, whereas the smoother regions originate from the stellar wind. The brightness contrast between the disk and the wind is $\sim 30$, but can reach 100 , if you compare the inner region of the disk with the outer parts of the wind. This 
Table 4. Best model parameters for the $\alpha$ Arae central star and its circumstellar environment obtained from this work. Values for the other parameters that have not changed are listed Table 1 and are from Paper I.

\begin{tabular}{cc}
\hline \hline Parameter/result & Value \\
\hline$v \sin i$ & $375 \mathrm{~km} \mathrm{~s}^{-1}$ \\
Inclination angle i & $55^{\circ}$ \\
Photospheric density $\left(\rho_{\text {phot }}\right)$ & $1.0 \times 10^{-11} \mathrm{~g} \mathrm{~cm}^{-3}$ \\
Equatorial rotation velocity & $470 \mathrm{~km} \mathrm{~s}^{-1}$ \\
Equatorial terminal velocity & $1 \mathrm{~km} \mathrm{~s}^{-1}$ \\
Polar terminal velocity & $1000 \mathrm{~km} \mathrm{~s}^{-1}$ \\
Polar mass flux & $7 \times 10^{-9} M_{\odot} \mathrm{year}^{-1} \mathrm{sr}^{-1}$ \\
$m 1$ & 0.5 \\
$m 2$ & 100.0 \\
$C 1$ & 0.03 \\
Mass of the disk & $4.1 \times 10^{-10} M_{\odot}$ \\
Mass loss & $1.3 \times 10^{-8} M_{\odot}$ year $^{-1}$ \\
\hline
\end{tabular}

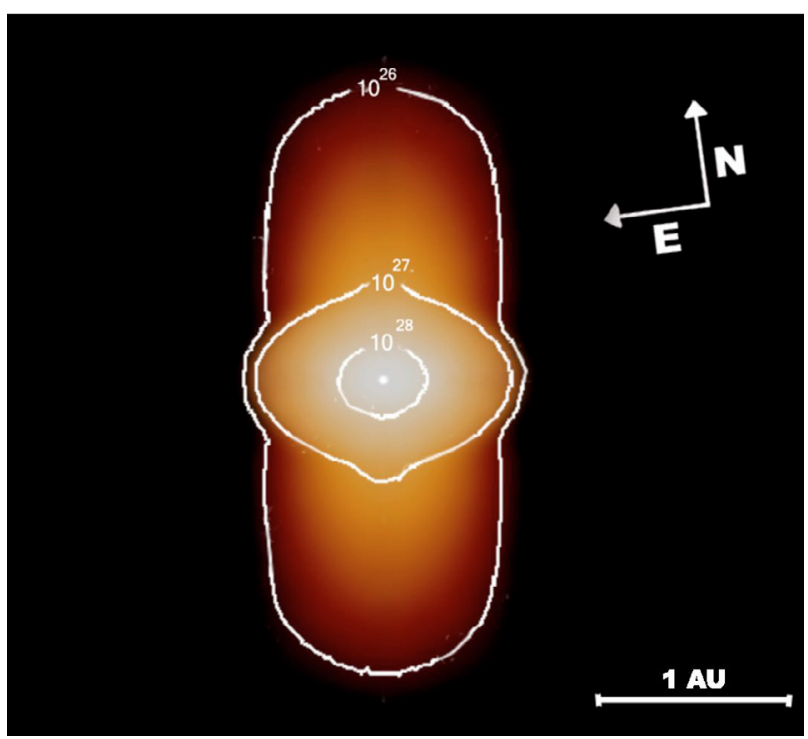

Fig. 8. Intensity map in the continuum at $2.15 \mu \mathrm{m}$ obtained with SIMECA for our best model parameters. The inclination angle is $55^{\circ}$, and the central bright region is the flux contribution from the thin equatorial disk, whereas the smoother regions originate in the stellar wind. The brightness contrast between the disk and the wind is globally $\sim 30$, but can reach 100, if you compare the inner region of the disk with the outer parts of the wind.

density contrast implies that we use a $C 1$ parameter smaller than 1 , i.e., 0.03 , to keep a sufficient equator/pole density contrast and a negligible equatorial expansion velocity $\left(1 \mathrm{~km} \mathrm{~s}^{-1}\right)$.

The fit of the visibility in the continuum basically constraints two parameters: the outer radius of the equatorial disk, which will modify the visibilities for all the projected baselines, and the inclination angle, which has a strong influence on the flattening of the projected equatorial disk into the skyplane. The outer radius of the equatorial disk for our best model was set to $33 R_{\star}$, which is larger than that in the $25 R_{\star}$ found in Paper I, but still in agreement with the $32 R_{\star}$ obtained for the companion orbit. Moreover, the inclination angle we obtain with our best model is $55 \pm 5^{\circ}$, which is also larger, but in agreement with the inclination angle of $45 \pm 5^{\circ}$ found in Paper I.

\subsection{Line profiles}

To put additional constraints on our modeling, we also try to fit emission line profiles with SIMECA, namely $\mathrm{H} \alpha, \mathrm{H} \beta$, and $\mathrm{Pa} \beta$.
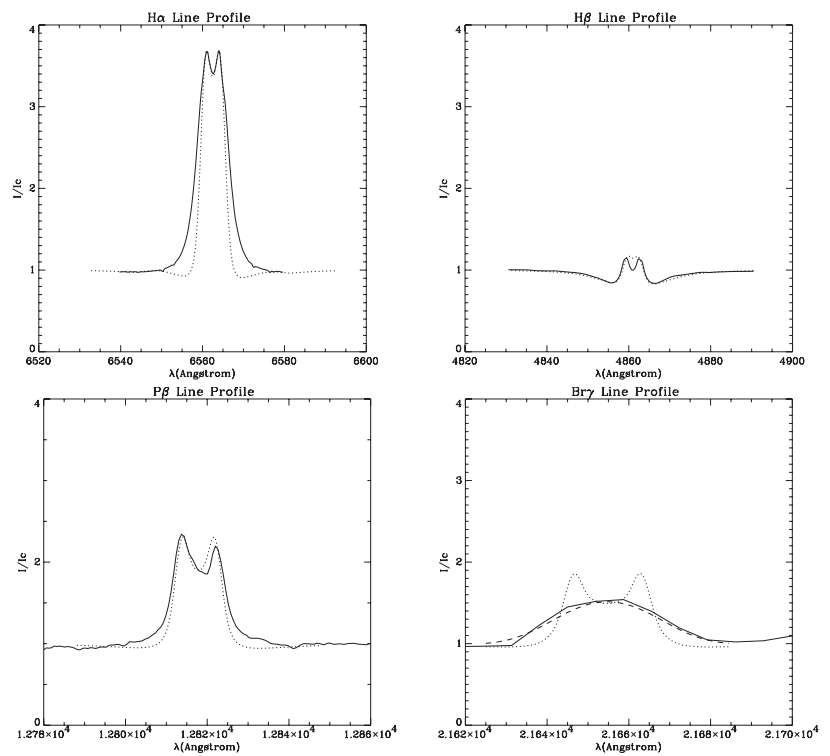

Fig. 9. Line profiles modeled with the SIMECA code using a thin disk + polar wind scenario. Modeled profiles are dotted lines, whereas observed ones are in solid lines. For $\operatorname{Br} \gamma$, the modeled profile (dotted line) obtain with SIMECA is convoluted with a $15 \AA$ Gaussian, corresponding to the AMBER spectral resolution of 1500. The convoluted $\mathrm{Br} \gamma$ profile we obtain is the dashed line superimposed on the observed one (solid line).

These lines were also used in Paper I, but following a quite different scenario: a nearly spherical expanding and rotating envelope versus a thin disk + polar wind, used in the present paper. Moreover, in this paper, we also used the $\operatorname{Br} \gamma$ emission line which is accessible to AMBER. We remind the reader that the $\mathrm{H} \alpha$ and $\mathrm{H} \beta$ line profiles were observed in April 1999, with the HEROS instrument at la Silla (Chile), the $\mathrm{Pa} \beta$ profile was recorded on August 13, 2003, at the Observatorio do Pico dos Dias (Brasil), and only the Br $\gamma$ line was contemporary to our AMBER interferometric observations. Knowing that $\alpha$ Arae is a variable star exhibiting line variations with typical timescales of a few months, we will only use the global shape of these line profiles to constrain the kinematics within the disk. We will not try to simultaneously fit the intensity of all these lines, which is, moreover, not possible, as shown in Paper I. In our new model, these emission lines originate from the dense and thin equatorial disk, and thus a fit of the shape of these lines will put strong constraints on the disk kinematics, since the disk is only supposed to rotate around the central star. Again, this is quite a different scenario from that in Paper I, where we used an expanding and rotating nearly spherical envelope to fit the lines.

The observed $\mathrm{Br} \gamma$ line profile does not show a double-peaked structure, due to the medium (1500) AMBER spectral resolution mode used for these first interferometric observations, since the high (10000) AMBER spectral resolution mode was not available at this time. Nevertheless, we can see in Fig. 9 that after a convolution with a $15 \AA$ Gaussian, corresponding to the AMBER spectral resolution, the agreement with the modeled profile and the AMBER ones is satisfying. For the other lines, the fit of the peak separation is in agreement with the Keplerian rotation. As already discussed in Paper I, we recall that between the $1999(\mathrm{H} \alpha$ and $\mathrm{H} \beta$ profiles), the $2003(\mathrm{~Pa} \beta)$, and the 2005 $(\operatorname{Br} \gamma)$ observations, we decreased the density at the base of the stellar photosphere by $25 \%$ to fit the line intensity. 




Fig. 10. Differential visibility across the $B r \gamma$ line profile for the $B_{0}, B_{1}$, $\mathrm{B}_{2}$, and $\mathrm{B}_{3}$ baselines. The first picture from the top is the $\mathrm{Br} \gamma$ line profile. The solid lines are the fits we obtain with SIMECA from our best models whereas the AMBER/VLTI data are the points with error bars.

Compared to Paper I, the agreement between the modeled and observed $\mathrm{H} \alpha$ line profiles in Fig. 9 is not as good. We are not able to reproduce the broad line wings, which were mainly due to the nearly spherical expanding stellar wind used in our previous model. In our new scenario, the geometrically thin and rotating equatorial disk produces a narrower $\mathrm{H} \alpha$ line profile. To obtain larger line widths, we should have taken into account multiple diffusion that occurs preferentially in the line wings, as shown by Poeckert \& Marlborough (1979). This is especially true for $\mathrm{H} \alpha$ and $\mathrm{Pa} \beta$, but less pronounced for $\mathrm{H} \beta$ and $\mathrm{Br} \gamma$. Nevertheless, we concentrate on the double-peaked separation, which is very sensitive to the rotational velocity law used, and as was already mentioned, the agreement we obtain was better using a Keplerian rotating law within the disk.

\subsection{Differential visibility modulus across the $\mathrm{Br} \gamma$ emission line}

The differential visibility curves are plotted in Fig. 10 for the $\mathrm{B}_{0}$, $\mathrm{B}_{1}, \mathrm{~B}_{2}$, and $\mathrm{B}_{3}$ baselines. The agreement between the modeled visibility and the AMBER/VLTI data is very good. Moreover, it was possible to reproduce the visibility decrease across the $\mathrm{Br} \gamma$ line profile, mainly due to the variation of the flux ratio between the unresolved star and the partially resolved circumstellar environment, but also due to its shapes as a function of wavelength. For the $\mathrm{B}_{2}$ baseline mainly along the equatorial disk direction, the $\mathrm{B}_{0}$ and $\mathrm{B}_{3}$ baselines close to the equatorial disk, the curve, with an overall " $U$ " shape, present a visibility increase at the center of the line. This means that $\alpha$ Arae observed within a narrow spectral bandwidth of $15 \AA$ appears smaller at the center of the emission line compared to its extension in the nearby emission line wings, as seen in Fig. 12. On the contrary, for the $\mathrm{B}_{1}$ baseline oriented along the polar axis the visibility curve presents a single "V" shape decrease at the line center. These
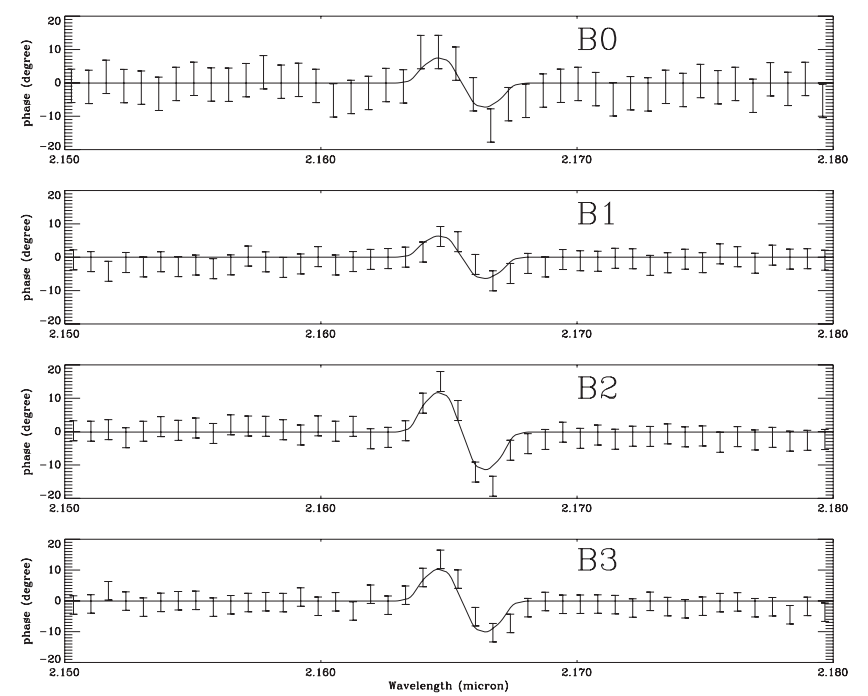

Fig. 11. Phase of the visibility across the $\mathrm{Br} \gamma$ line profile for the $\mathrm{B}_{0}$, $\mathrm{B}_{1}, \mathrm{~B}_{2}$, and $\mathrm{B}_{3}$ baselines. The solid lines are the fits we obtain with SIMECA from our best model, whereas the AMBER/VLTI data are the points with error bars.

effects are mainly due to the kinematics within the disk and are confirmed by the shape of the phase of the visibility, as we will see in the following section.

\subsection{Differential phase across the Bry emission line}

The shape of the differential phase across the $\operatorname{Br} \gamma$ line profile is related to the photocenter displacement of the object as a function of wavelength and is very sensitive to the $\beta$ law used in the modeling, as already shown by Stee (1996). For our best model, we used a Keplerian rotation within the disk and the resulting visibility phases as a function of wavelength for the $\mathrm{B}_{0}, \mathrm{~B}_{1}, \mathrm{~B}_{2}$, and $\mathrm{B}_{3}$ baselines, are plotted Fig. 11. Again, the agreement between the modeled phases and the AMBER/VLTI data is very good, which is the first direct evidence of the Keplerian rotation within a Be circumstellar disk. Nevertheless, to know if this agreement is unique, we have tested different models with different disk rotational velocities following:

$v_{\phi}(r, \theta) \propto \sin \theta\left(\frac{R}{r}\right)^{\beta}$,

with $\beta$ between the constant rotation $(\beta=0)$ and the angular momentum conservation $(\beta=1.0)$, respectively $\beta=0,0.3,0.4,0.45$, $0.5,0.55,0.7$, and 1 . Nevertheless, if you modify the $\beta$ law and still want to fit the doublepeaks of the $\mathrm{Br} \gamma$ emission line profile, you need to simultaneously modify the equatorial rotational velocity of the star, since the inner part of the disk, supposed to be in contact with the star's photosphere, rotates at the same velocity. Thus, we were obliged to take into account the shape of the star photosphere and its distortion due to its fast rotational velocity, as shown by Domiciano de Souza et al. (2003) for the Be star Achernar, which was rotating close to its critical velocity, and thus exhibits a flattened photosphere with an equatorial vs. polar radius ratio of 1.5 . In Fig. 13 we plotted the "beta" law versus the equatorial rotational velocity that fit the doublepeaks of the $\mathrm{Br} \gamma$ line emission line profile, assuming a purely circumstellar rotating disk without expansion. This figure shows that if we assume that the stellar rotation is between $v_{\min }$, corresponding to the smaller measured $v \sin i=288 \mathrm{~km} \mathrm{~s}^{-1}$ by Uesugi \& Fukuda (1970), with $i=55^{\circ}$, i.e., $v_{\min }=350 \mathrm{~km} \mathrm{~s}^{-1}$, and $v_{\max }$, 


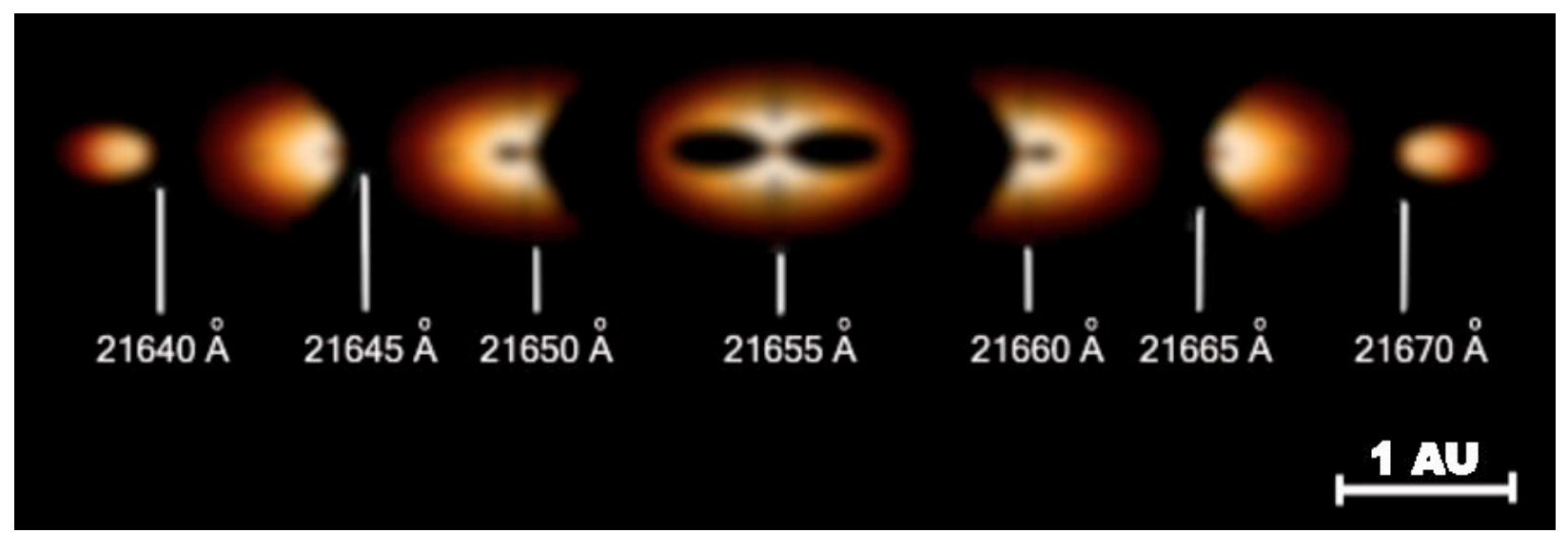

Fig. 12. Intensity maps across the $\operatorname{Br} \gamma$ line profiles within spectral channels of $15 \AA$, from which the differential visibility modulus and phase are estimated. Note that to increase the image contrast, the central star and the continuum emission have been subtracted.

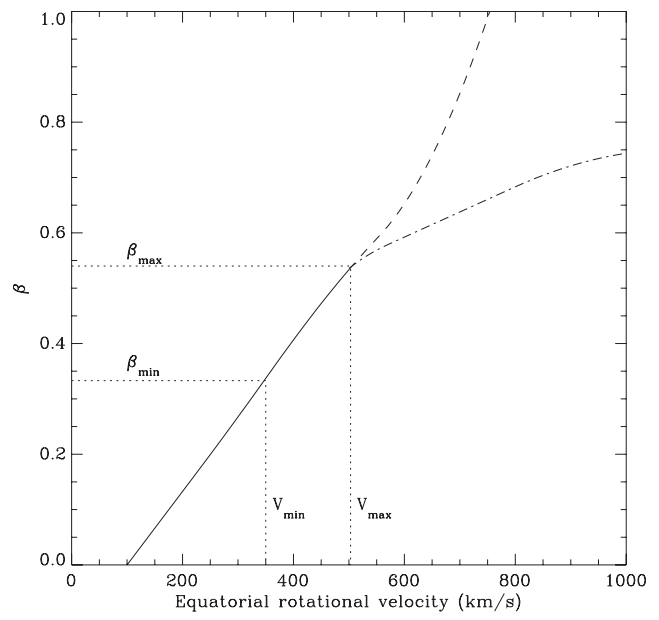

Fig. 13. $\beta$ parameter as a function of the equatorial velocity, taking into account the stellar photosphere deformation due to its fast rotation. The solid line corresponds to physically possible cases, whereas the dashed line corresponds to rotational values larger than the "breakup" velocity. The dash-dotted line also corresponds to unrealistic values, but in this case we have not taken into account the stellar photosphere distortion larger than $1.5 R_{\star}$ at the equator. $v_{\min }=351 \mathrm{~km} \mathrm{~s}^{-1}$ corresponds to the minimum vsini found in the literature for this star, i.e., $288 \mathrm{~km} \mathrm{~s}^{-1}$, by Uesugi \& Fukuda (1970) with $i=55^{\circ}$.

corresponding to the critical velocity for this star, i.e., $v_{\max }=$ $503 \mathrm{~km} \mathrm{~s}^{-1}$; the $\beta$ parameter must lie between 0.33 and 0.54 . This last value is in better agreement with the upper value of the measured vsini, $375 \mathrm{~km} \mathrm{~s}^{-1}$, by Bernacca \& Perinotto (1970) with $i=55^{\circ}$, i.e., $v=457 \mathrm{~km} \mathrm{~s}^{-1}$ at the equator which, may be a good indication that $\alpha$ Arae is rotating very close to its critical velocity.

Moreover, to be more quantitative, we have computed the total $\chi^{2}$ from our best model as a function of the $\beta$ parameter. The results are plotted Fig. 14 and we clearly see that better agreement $\left(\chi_{\min }^{2}\right)$ is obtained for a rotating law very close to the Keplerian rotation, i.e., $\chi_{\min }^{2}=0.48 \pm 0.04$.

\subsection{Is the geometrically thin and rotating disk scenario unique?}

Since our Keplerian disk + polar enhanced winds scenario seems to successfully reproduce all the available observables (i.e.,

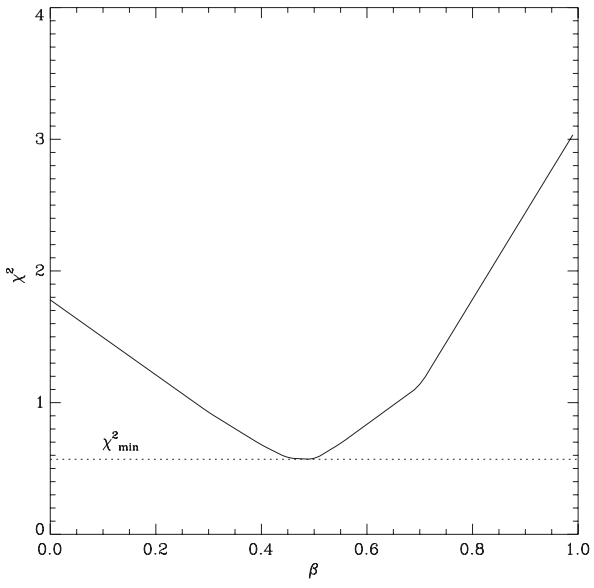

Fig. 14. $\chi^{2}$ from our models and the AMBER data plotted as a function of the $\beta$ parameter. $\chi_{\min }^{2}$ is the minimum value obtained for a model with $\beta=0.48$, which is very close to the Keplerian rotation $(\beta=0.5)$.

photometric, spectroscopic, polarimetric, and interferometric) we may wonder if this scenario is really unique, especially due to the number of parameters used in the SIMECA code. Thus, we have tested two other scenarios; the first one is based on the same global geometry of our best model, but we have now added an expansion component produced by an equatorial stellar wind.

Since the peak separation of the line profiles depends on both components of the velocity field, i.e., expansion and rotation, we have to decrease the rotational velocity used in our best model to keep the same observed peak separation. Remembering that the lowest possible value of the rotational velocity is $351 \mathrm{~km} \mathrm{~s}^{-1}$, we can only use a maximum value of $100 \mathrm{~km} \mathrm{~s}^{-1}$ for the expansion equatorial terminal velocity. The corresponding differential visibilities and phases we obtain are plotted in Fig. 15. In this case, the decrease of the differential phase amplitude variations is due to the smaller value used for the rotating component. If the envelope was only in spherical expansion due to a spherical stellar wind, the phase variation across the $\operatorname{Br} \gamma$ line would be zero, since there is no photocenter displacement for a spherically symmetric velocity field. On the contrary, if you have a purely rotating disk, the photocenter displacement will follow the projection of the isovelocity regions and will produce a typical "S-shape", as shown Fig. 11. Thus, a decrease of the rotational velocity field component regarding the 

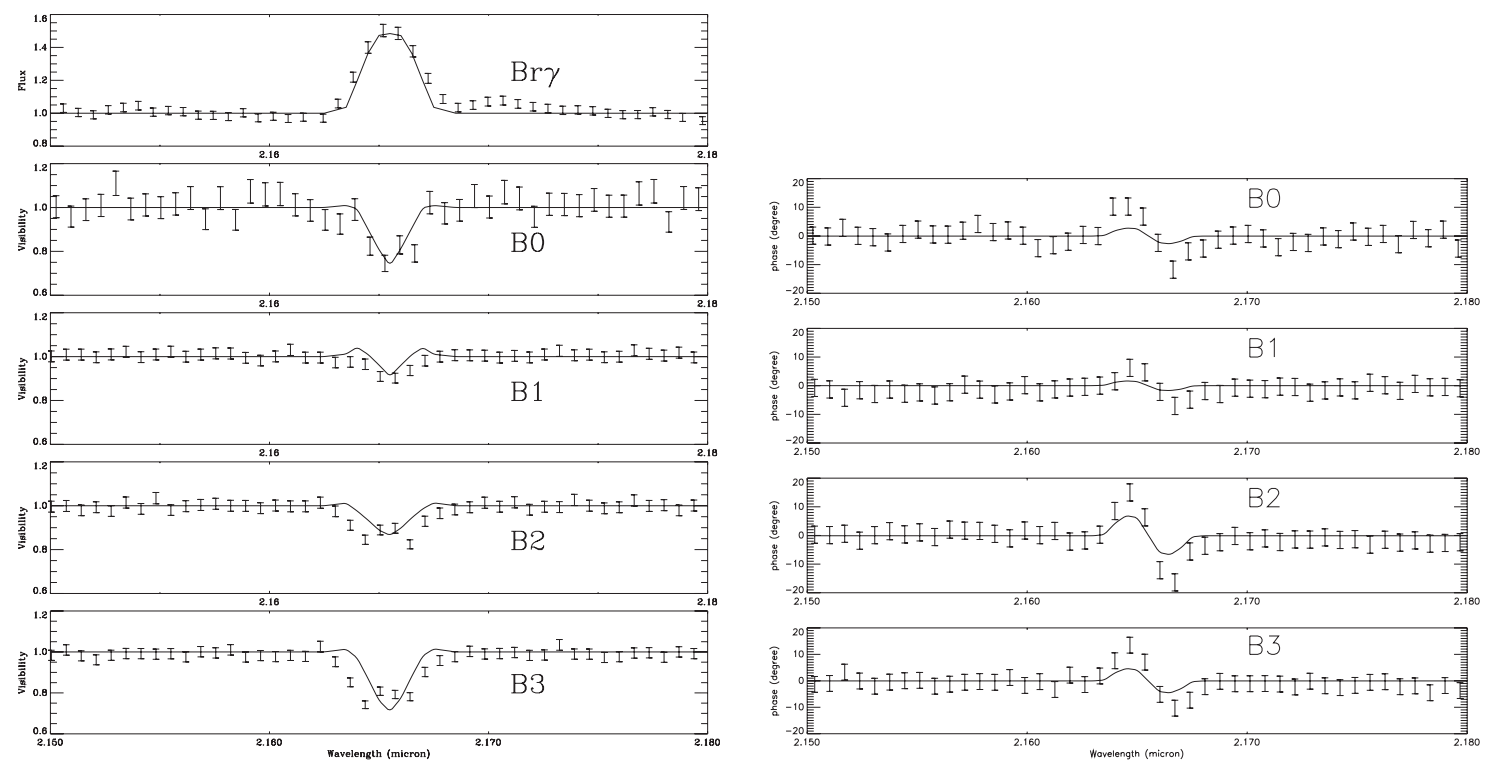

Fig. 15. $\alpha$ Arae differential visibilities (left) and phases (right) across the $\mathrm{Br} \gamma$ line profiles for the $\mathrm{B}_{0}, \mathrm{~B}_{1}, \mathrm{~B}_{2}$, and $\mathrm{B}_{3}$ baselines. The solid lines are the fits we obtain with SIMECA from a rotating and expanding scenario described in Sect. 6.6, whereas the AMBER/VLTI data are the points with error bars.
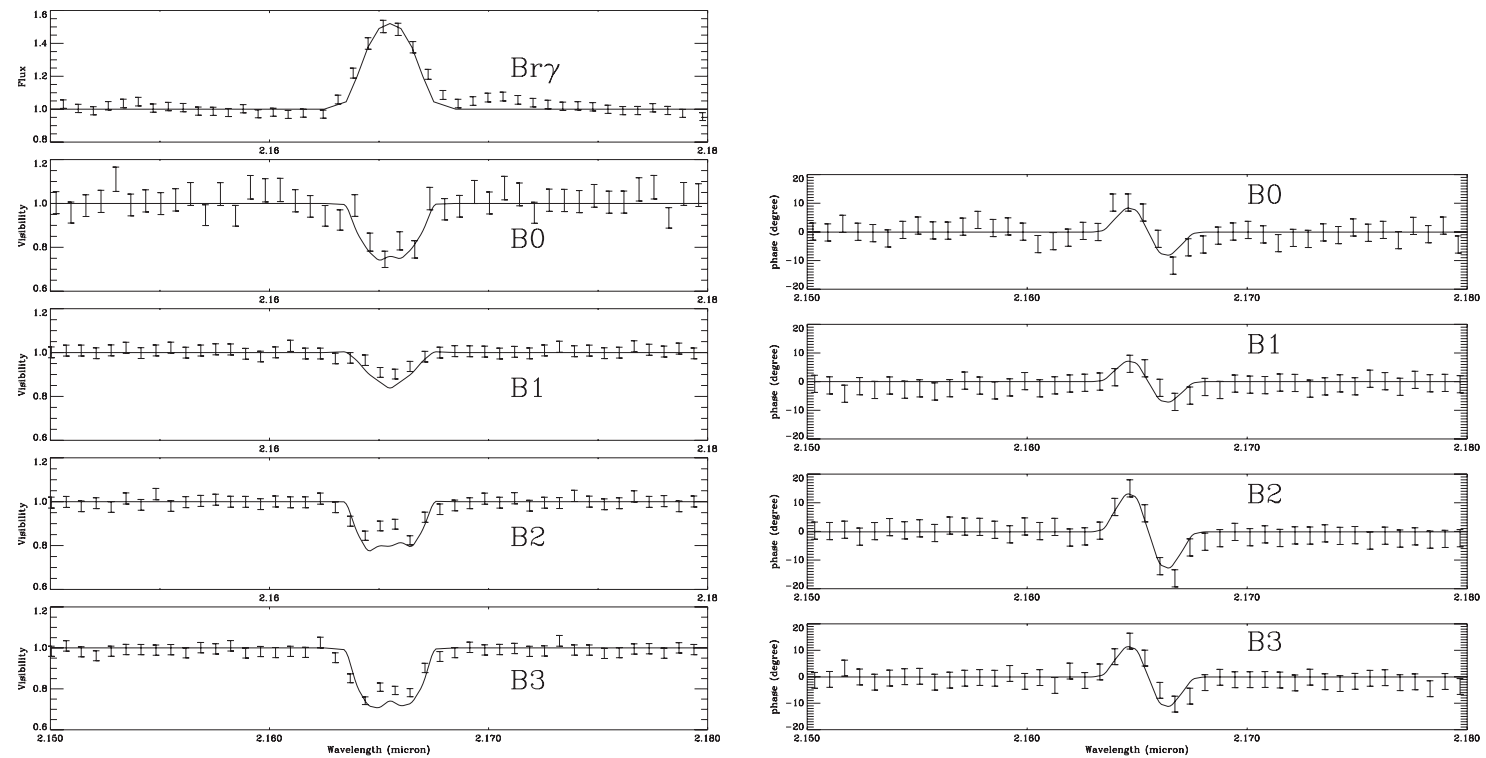

Fig. 16. $\alpha$ Arae differential visibilities (left) and phases (right) across the $\mathrm{Br} \gamma$ line profiles for the $\mathrm{B}_{0}, \mathrm{~B}_{1}, \mathrm{~B}_{2}$, and $\mathrm{B}_{3}$ baselines. The solid lines are the fits we obtain with SIMECA from the model 3 described in Sects. 4.3.2 and 6.6, whereas the AMBER/VLTI data are the points with error bars.

expansion component globally produces the smaller differential phase amplitude variation shown in Fig. 14. The amplitude of the differential visibility across the line remains almost the same, since it is less sensitive to the kinematics within the disk, but more sensitive to a global geometric change of the circumstellar environment, to a change in the star versus envelope flux ratio in the line, and to the continuum which remains unchanged by modifying the velocity fields. Nevertheless, the shape of the differential visibility is strongly modified and now presents a "V" shape, as was already mentioned in Sect. 6.4. This shape variation is due to the fact that the expansion versus rotation ratio is increasing in the equatorial region, and the difference between the pole versus equator kinematics is less pronounced when adding an equatorial expansion component. This analysis exhibits the importance of spectrally resolved interferometric measurements for the study of the kinematics within circumstellar disks.
The second scenario is based on a quasi-spherical model like the model 3 already described in our "toy story" section. The kinematics used are very close to our thin disk + polar enhanced winds model, (i.e., Keplerian rotation with a $1 \mathrm{~km} \mathrm{~s}^{-1}$ equatorial terminal velocity), but in this later case the disk is rather geometrically thick. Again, the corresponding differential visibilities and phases we obtain are plotted in Fig. 15. Since the kinematics remains mostly unchanged, the fits of the differential phases are as good as for our best model, but since the geometry of the disk is different, the agreement with the differential visibilities is not as good. Again, thanks to the same disk kinematics, the shape of the differential visibilities is very similar, but their amplitudes are not well reproduced. Nevertheless, these differences remain very small for an inclination angle of $55^{\circ}$, and it is very hard to put an upper limit for the disk opening angle using this method, especially regarding the actual errors on the AMBER absolute calibrated visibilities. On the other hand, this 
study clearly shows that the equatorial region is a Keplerian rotating disk rather than an expanding wind.

\section{Discussion}

This study points out three important results touching lively debated issues:

\subsection{Keplerian rotation}

There were already some indications by other theoretical studies that the disk may follow the Keplerian rotation, for instance the results obtained by Hanuschik (1995) regarding shell lines produced within a Keplerian disk in hydrostatic equilibrium. Hanuschik $(1996,2000)$ also investigates the geometrical structure of the emitting part of circumstellar envelopes around Be stars and found a good agreement with spectroscopic data, using a thin disk in vertical hydrostatic and horizontal centrifuginal equilibrium, similar to a Keplerian accretion disk. These results were confirmed by Rivinius (1999), presenting high resolution echelle spectra for 6 B-type stars that were supposed to be seen edge-on, and in good agreement with Hanuschick's models for the formation of shell lines in circumstellar disks with Keplerian rotation. In a more recent paper, Rivinius (1999) proposes a scenario where the disk is no longer a completely stationary structure, but rather a succession of outbursts that may form rings. But even within this scenario, part of the ejected matter attains a sufficiently high angular momentum to form a roughly Keplerian disk, at least for the immediate period following the outbursts. Finally, another indirect argument in favor of a Keplerian disk is the success of the global oscillation modeling already outlined in the paper review by Porter \& Rivinius (2003). Thus, our results may confirm the widely accepted fact that circumstellar disks around Be stars are in Keplerian rotation.

\subsection{Stellar rotation}

As already mentioned in Sect. 6.5 we found that $\alpha$ Arae must be rotating very close to its critical velocity, since we obtain $\frac{v_{\text {rot }}}{v_{\text {crit }}} \sim 91 \%$. This value is far above the conservative estimates of $\sim 75 \%$ usually found in the literature for Be stars. The fact that Be stars may be rotating much closer to their critical velocities than is generally supposed was already outlined by Townsend et al. (2004) and Owocki (1996). This nearly critical rotation has quite profound implications for dynamical models of Be disk formation and may be the clue for the Be phenomenon. It may bring sufficient energy to levitate material in a strong gravitational field, or at least help other physical processes such as pulsation or gas pressure provide sufficient energy and angular momentum to create a circumstellar disk. Moreover, observational evidence of this nearly critical rotation are growing, such as the results obtained by Domiciano et al. (2003) using interferometric VINCI/VLTI data of Achernar. They measured a rotationally distorted photosphere with an apparent oblateness of 1.56, which cannot be explained using the classical Roche approximation. This scenario follows the original picture by Struve (1931) of a critically rotating star, ejecting material from its equatorial regions.

\subsection{Polar wind enhancement}

Our interferometric measurements shows evidence of a polar wind enhancement (see Fig. 8) that was already predicted for almost critically rotating stars. For instance, Cranmer \& Owocki (1995) and Owocki \& Gayley (1998) studied the effects of limb darkening, gravity darkening, and oblateness on the radiation driving mechanism, and found that the tendency for the higher flux from the bright poles to drive material toward the darker equatorial region is outweighed by the opposite tendency for the oblateness of the stellar surface to direct the radiative flux to higher latitudes, i.e., away from the equator. The paper review by Porter \& Rivinius (2003) also outlines the effect of the inclusion of nonradial line-driving force which reduces the effect of the wind compression to zero and, taking into account the gravity darkening, results in a polar wind enhancement. This physical effect goes in the opposite direction to the one proposed for the Wind Compressed Disk model by Bjorman \& Cassinelli (1993). In a recent paper, Kervella \& Domiciano de Souza (2006) have shown an enhanced polar wind for the Be star Achernar, whereas this Be star presents no hydrogen lines in strong emission. Thus, it seems that a significant polar wind may be present, even if the star is still in a normal B phase, i.e., this enhanced polar wind does not seem to be related to the existence of a dense equatorial envelope, as already outlined by Kervella \& Domiciano de Souza (2006).

\section{Conclusion}

1. Thanks to these first spectrally resolved interferometric measurements of a Be star at $2 \mu \mathrm{m}$, we are able to propose a possible scenario for the Be star $\alpha$ Arae's circumstellar environment, which consists of a thin disk + polar enhanced winds that is successfully modeled with the SIMECA code.

2. We found that the disk around $\alpha$ Arae is compatible with a dense equatorial matter confined in the central region, whereas a polar wind is contributing along the rotational axis of the central star. Between these two regions, the density must be low enough to reproduce the large visibility modulus (small extension) obtained for two of the four VLTI baselines. This new scenario is also compatible with the previous MIDI measurements and the fact that the outer part of the disk may be truncated by a unseen companion at $32 R_{\star}$.

3. For the first time, we obtain the clear evidence that the disk is in Keplerian rotation, closing a debate that has continued since the discovery of the first Be star $\gamma$ Cas by Father Secchi in 1866.

4. We found that $\alpha$ Arae must be rotating very close to its critical velocity.

5. These observations were done using the medium (1500) spectral resolution of the AMBER/VLTI instrument and are very promising for the forthcoming AMBER high spectral resolution observational mode (10000) and the coupling of the Auxiliary Telescopes (ATs) on the VLTI array.

Acknowledgements. We thanks G. Duvert and J.-M. Clausse for their help in the data reduction through the JMMC. We acknowledge the useful comments of the referee Thomas Rivinius who help to improve the discussion in this paper. The preparation and interpretation of AMBER observations benefit from the tools developed by the Jean-Marie Mariotti Center for optical interferometry (JMMC) and from the databases of the Centre de Données Stellaires (CDS) and of the Smithsonian/NASA Astrophysics Data System (ADS). The data reduction software amdlib and the user-friendly interface ammyorick used in this work is available on the AMBER site http://amber.obs.ujf-grenoble.fr.

\section{References}

Araùjo, F. X., \& Freitas Pacheco, J. A. 1989, MNRAS, 241, 543

Bernacca, P. L., \& Perinotto, M. 1970, A catalogue of stellar rotational velocities, Contr. Oss. Astroph. Padova in Asiago, 239, 1 
Bjorkman, J. E., \& Cassinelli, J. P. 1993, ApJ, 409, 429

Bjorkman, K. S., Nook, M. A., Nordsiek, K. H., et al. 1997, ApJ, 477, 926

Bjorkman, J. E., \& Carciofi, A. C. 2004, A\&AS, 204, 6206

Castor, J. I., Abbott, D. C., \& Klein, R. I. 1975, ApJ, 195, 157

Chauville, J., Zorec, J., Ballereau, D., et al. 2001, A\&A, 378, 861

Chesneau, O., Meilland, A., Rivinius, T., et al. 2005, A\&A, 435, 275 (Paper I)

Cohen, D. H., Cassinelli, J. P., \& MacFarlane, J. J. 1997, ApJ, 487, 867

Cranmer, S. R., \& Owocki, S. P. 1995, ApJ, 440, 308

Domiciano de Souza, A., Kervella, P., Jankov, S., et al. 2003, A\&A, 407, L47

Dougherty, S. M., Waters, L. B. F. M., Burki, G., et al. 1994, A\&A, 290, 609

Ducati, J. R. 2002, Stellar Photometry in Johnson's 11-color system, Catalogue

of Stellar Photometry in Johnson's 11-color system

Hanuschik, R. W. 1995, A\&A, 423, 434

Hanuschik, R. W. 1996, A\&A, 308, 170

Hanuschik, R. W. 2000, ASPC, 214, 518

Hubeny, I. 1988, Comp. Phys. Comm., 52, 103

Hubeny, I., \& Lanz, T. 1995, ApJ, 439, 875

Jamar, C., Macau-Hercot, D., Monfils, A., et al. 1976, UV Bright Star Spectrophotometric Catalog, ESA SR-27

Johnson, H. L., Mitchell, R. I., Iriarte, B., \& Wisniewski, W. Z. 1966, UBV Photometry of Bright Stars, Commun. Lunar \& Planet. Lab. 4, 99, Table 9

Kervella, P., \& Domiciano de Souza, A. 2006, A\&A, 453, 1059

Mc Lean, I. S., \& Clarke, D. 1979, MNRAS, 186, 245

Owocki, S. P. 2005, ASPC, 337, 101

Owocki, S. P., \& Gayley, K. G. 1998, ASPC, 131, 237

Owocki, S. P., Cranmer, S. R., \& Blondin, J. M. 1994, ApJ, 424, 887

Owocki, S. P., Cranmer, S. R., \& Gayley, K. G. 1996, ApJ, 472, L115
Perryman, M. A. C., Lindegren, L., Kovalevsky, J., et al. 1997, A\&A, 323, 49 Petrov, R. G., Malbet, F., Weigelt, G., et al. 2007, A\&A, 464, 1

Poe, C. H., \& Friend, D. 1986, ApJ, 311, 317

Poeckert, R., \& Marlborough, J. M. 1979, ApJ, 233, 259

Porter, J. M., \& Rivinius, Th. 2003, PASP, 115, 1153

Quirrenbach, A., Bjorkman, K. S., Bjorkman, J. E., et al. 1997, ApJ, 479, 477

Richichi, A., \& Percheron, I. 2002, A\&A, 386, 492

Rivinius, Th., Stefl, S., \& Baade, D. 1999, A\&A, 348, 831

Rivinius, Th., Baade, D., Stefl, S., et al. 2001, A\&A, 379, 257

Secchi, A. 1867, Astron. Nachr., 68, 63

Stee, Ph. 1996, A\&A, 311, 945

Stee, Ph. 2003, A\&A, 403, 1023

Stee, Ph., \& de Araùjo, F. X. 1994, A\&A, 292, 221

Stee, Ph., \& Bittar, J. 2001, A\&A, 367, 532

Stee, Ph., de Araùjo, F. X., Vakili, F., et al. 1995, A\&A, 300, 219

Stee, Ph., Vakili, F., Bonneau, D., et al. 1998, A\&A, 332, 268

Struve, O. 1931, ApJ, 73, 94

Tatulli, E., Millour, F., Chelli, A., et al. 2007, A\&A, 464, 29

Thomson, G. I., Nandy, K., Jamar, C., et al. 1978, Catalogue of stellar ultraviolet fluxes, The Science Research Council, UK

Uesugi, A., \& Fukuda, I. 1970, Catalogue of rotational velocities of the stars, Contr. Astroph. Kwasan, Obs. Univ. Kyoto, 189

Townsend, R. H., Owocki, S. P., \& Howarth, I. D. 2004, MNRAS, 350, 189

Wood, K., Bjorkman, J. E., Whitney, B. A., \& Code, A. D. 1996a, ApJ, 461, 828

Wood, K., Bjorkman, J. E., Whitney, B. A., \& Code, A. D. 1996b, ApJ, 461, 847

Yudin, R. V., \& Evans, A. 1998, A\&AS, 131, 401

Yudin, R. V. 2001, A\&A, 368, 912 